\title{
Delayed Avalanches in Multi-Pixel Photon Counters
}

\author{
Fabrice Retière ${ }^{1}$ \\ TRIUMF \\ 4004 Wesbrook Mall, Vancouver, BC V6T 2A3, Canada \\ E-mail: fretiere@triumf.ca
}

Kyle Boone

University of British Columbia

Department of Physics and Astronomy, 6224 Agricultural Road, Vancouver, BC V6T 1Z1, Canada

\begin{abstract}
Hamamatsu Photonics Multi-Pixel Photon Counters (MPPCs) achieve high photo-detection efficiency with relatively low dark noise. The MPPC gain is comparable to Photo-multipliers but pixel avalanches tend to trigger others either in neighbouring pixels (cross-talk) or in the same pixel at a later time (after-pulsing). We investigate the possibility that after-pulsing avalanches are due to visible photons created in the avalanches and absorbed in the silicon bulk. In this case, the charge carriers created in the silicon bulk may diffuse back to the high field region, producing delayed avalanches. The MPPCs were illuminated with laser light at wavelengths ranging from 404 to $820 \mathrm{~nm}$ and the timing distribution of the avalanches was measured. Delayed avalanches were observed at 637 and $820 \mathrm{~nm}$ showing that some holes do diffuse to the high field region. Simulations show that optical photons are responsible for at least half of the after-pulsing avalanches.
\end{abstract}

International Workshop on New Photon-detectors,

LAL Orsay, France

June 13-15, 2012

\footnotetext{
$1 \quad$ Speaker
} 


\section{Introduction}

Hamamatsu Photonics Inc. is one of the leading manufacturers of pixilated Geiger-mode avalanche photo-diodes, so-called Silicon Photo-multipliers (SiPMs) under the brand name Multi-Pixel Photon Counter (MPPC) [1]. MPPCs achieve a high photo-detection efficiency in the blue ( 35\%) and low dark noise but they suffer from a significant rate of correlated avalanches that limit their operating voltage range[2,3]. MPPCs are typically operated between 0.5 and $2 \mathrm{~V}$ over the breakdown voltage where Geiger Mode avalanche start. Above $2 \mathrm{~V}$, the rate of correlated avalanches is so large that dark noise can trigger very large pulses ( $>10$ photoelectrons equivalent) and the energy and timing resolution start to worsen [2,4]. In 2011, it had become obvious that to improve MPPC performances, one would have to lower the correlated avalanche rate.

Correlated avalanches are often divided into two processes: cross-talk and after-pulsing. In this paper we will use an experimentally motivated definition for cross-talk and after-pulsing: cross-talk includes all the correlated avalanches that occur within a nanosecond of the parent, while after-pulsing includes any avalanches occurring after $1 \mathrm{~ns}$ [5]. Cross-talk is thought to be caused by visible photons produced in the avalanche that are absorbed in neighboring micro-cell [6]. Charge carriers trapped by impurities during avalanches and released at a later time are expected to be the cause of after-pulsing [7]. The rate of after-pulsing was found to be about double the cross-talk rate in [2].

Dark noise and after-pulsing inducing impurities are likely related, if not the same. However, Hamamatsu has successfully reduced dark noise by improving its manufacturing process, especially by reducing the concentration of impurities but it had little effect on the after-pulsing rate. We decided to investigate the possibility that after-pulsing could also be due to visible photons, with the delay between the parent and the daughter avalanches being the time taken by the charge carriers to reach the high field region. Indeed, charge carriers, holes in the case of MPPCs, created by the absorption of photons in the silicon bulk would have to diffuse to reach the high field region. The diffusion time appears to be consistent with the measured timing distribution of after-pulsing. In order to test this hypothesis, we designed an experiment using several wavelengths to vary the photon absorption depth. A simulation code was developed to interpret the data.

\section{Experiment}

The energy spectra of photons emitted in MPPC avalanches has been measured in [8]. Photons can produce electron-hole pairs in Silicon for wavelengths lower than about 1100nm. External light sources of variable wavelengths can be used to investigate whether or not holes created by photon absorption in the silicon bulk can trigger avalanches. We used 4 different laser heads using a Hamamatsu PLP-10 pico-second light source (with the attenuation lengths in Si): $405 \mathrm{~nm}(0.12 \mu \mathrm{m}), 467 \mathrm{~nm}(0.55 \mu \mathrm{m}), 637 \mathrm{~nm}(3.2 \mu \mathrm{m})$, and $820 \mathrm{~nm}(14.1 \mu \mathrm{m})$. The $820 \mathrm{~nm}$ laser system was lent to us by Hamamatsu. The MPPC high field region is expected to be 2$5 \mu \mathrm{m}$ deep below the Silicon surface. Hence, the 405 and 467nm are expected to trigger only 
prompt avalanches, while delayed avalanches should be visible at $637 \mathrm{~nm}$ and $820 \mathrm{~nm}$ if holes from the bulk can diffuse back to the high field region.

The tested MPPCs were specifically designed for the T2K experiment. They have 667 micro-cells ( $50 \mu \mathrm{m}$ pitch) for an active area of $1.3 \times 1.3 \mathrm{~mm} 2$. They are very similar to 400 microcells, $1 \times 1 \mathrm{~mm} 2$ MPPCs.

The laser light was attenuated using Ocean Optics digital variable attenuator to produce about 0.15 avalanches on average. Data were acquired over $1 \mu$ s using a digital oscilloscope. The trigger was set so that the prompt laser light would be recorded at 200ns within the acquisition window. A pulse finder was used to identify the avalanches in waveforms. The events with more than one avalanche were discarded in order to avoid having to deal with after-pulses. Figure 1 shows the timing distribution of the avalanches at the 4 different wavelengths. Dark noise was subtracted out from the timing distribution by measuring its contribution in the first $200 \mathrm{~ns}$ of the waveforms. It is indeed constant throughout the $1 \mu \mathrm{s}$ window. The data shown in Figure 1 were taken at $-60^{\circ} \mathrm{C}$ because at room temperature the dark noise rate is comparable to the delayed avalanche rate beyond about 20ns.

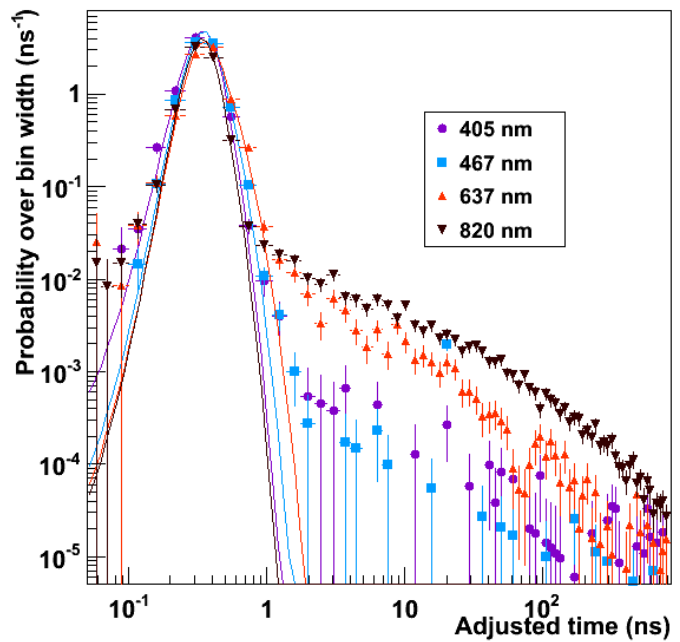

Figure 1. Timing distribution of avalanches produced by different laser wavelengths at $-60^{\circ} \mathrm{C}$ and $1.9 \mathrm{~V}$ over-voltage. The prompt peak was set to 0.3 ns in order to show well on the logarithmic plot.

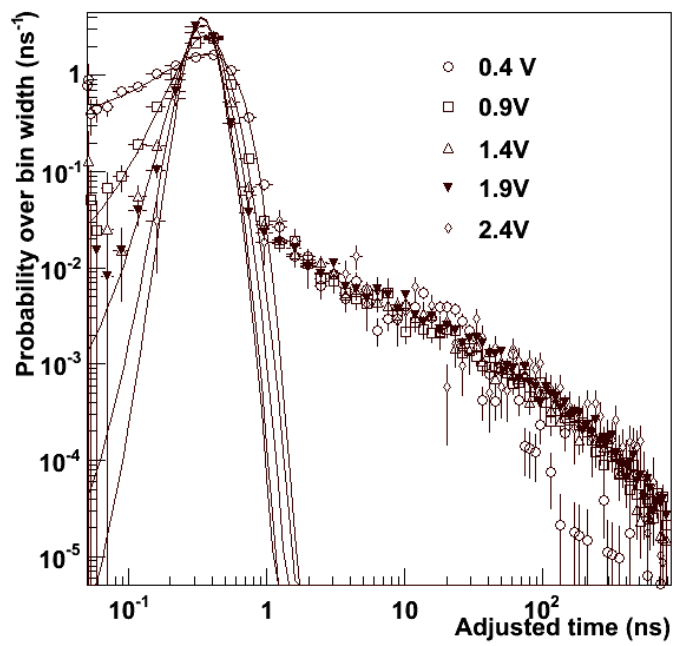

Figure 2. Timing distribution for different overvoltages at $-60 \mathrm{C}$ and for $820 \mathrm{~nm}$ wavelengths.

Figure 1 clearly shows delayed avalanches at $637 \mathrm{~nm}$ and $820 \mathrm{~nm}$ but none at $405 \mathrm{~nm}$ and $467 \mathrm{~nm}$. This result is consistent with expectations for delayed avalanches caused by holes diffusing from the bulk to the high field region. Figure 2 shows the dependence of the timing distribution with over-voltage. The prompt peak width clearly narrows with increasing overvoltage. On the other hand, the distribution of the tail changes little. This is expected because the conditions in the bulk are unaffected by what is happening around the high field region.

Not so obvious in Figure 2 is the fact that the relative probability of prompt versus late avalanches changes with over-voltage. Figure 3 shows the fraction of prompt avalanches as a function of over-voltage for 3 different wavelengths. There is a clear decreasing trend at $637 \mathrm{~nm}$ and $820 \mathrm{~nm}$, which again suggests that holes are responsible for delayed avalanches. Indeed, the impact ionization probability for holes is significantly smaller than for electrons, which means 
that holes require a higher field, i.e. a higher over-voltage than electrons to trigger avalanches [9]. In other words, the relative contribution of hole triggered avalanches increase with increasing over-voltage unless the photons are all absorbed before reaching the $\mathrm{n}+$ region.

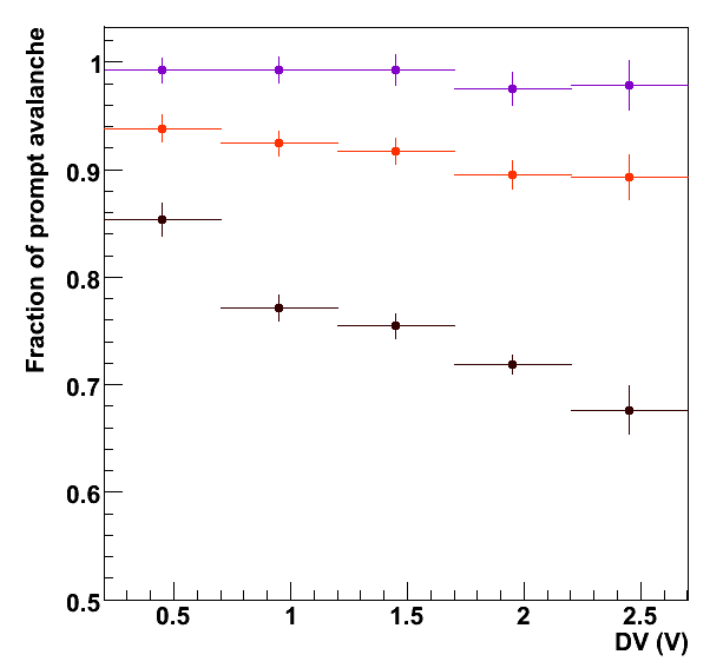

Figure 3. Fraction of prompt avalanches as a function of over-voltage for 3 different wavelengths: $405 \mathrm{~nm}$ (violet), 637nm (red) and 820nm (brown).

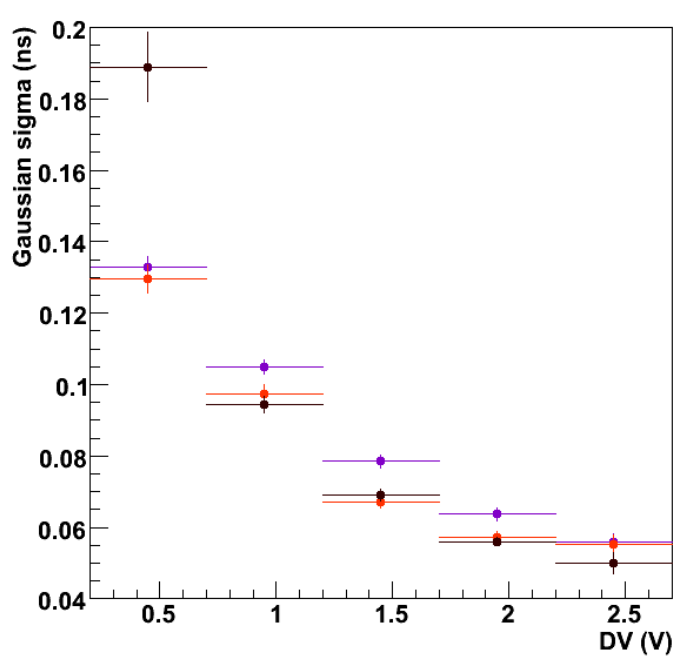

Figure 4. Width of the prompt peak ( $\sigma$ of a Gaussian distribution) as a function of overvoltage for 3 different wavelengths: $405 \mathrm{~nm}$ (violet), 637nm (red) and 820nm (brown).

The prompt peak is fitted by the convolution of a Gaussian distribution with an exponential function accounting for delayed avalanches within the first ns. The source of such delayed avalanches is different than the one discussed earlier, the time scales being very different. The prompt exponential time constant is indeed between 60 and 150ps. The origin of this "fast" tail is not known. The primary carriers must originate from a region of low or zero field close to the high field region. Figure 4 shows the $\sigma$ of the Gaussian distribution, which would be the single photon timing resolution (SPTR) if the exponential tail was absent. Overall, our timing resolution measurements are in good agreement with [4].

\section{Interpretation by simulations}

The data suggests that holes diffusing from within the bulk can trigger avalanches. In order to verify this hypothesis, we developed a simulation that could be directly compared to the data. The simulations have few free parameters because the properties of Silicon such as the light attenuation lengths and diffusion constants are well known. The free parameters are the hole life time in the bulk and the detailed structure of the junction that Hamamatsu keeps secret. In particular the junction depth is a key parameter that has to be determined from the data. A schematic drawing of the micro-cell structure used in the simulations is shown in Figure 5. The shape of the prompt peak is Gaussian with an exponential tail showing that some avalanches are delayed by 100 to 300ps, which is too fast to be caused by hole diffusion. Instead, low field collection regions were included in the simulations in order to account for the slower exponential tail. Figures 6 and 7 show comparisons of the data and simulations at 637 and $820 \mathrm{~nm}$. The same parameters were used for both simulations. The data are well reproduced by 
the simulations. The hole lifetime that best fit the data is 300ns, which is reasonable. The simulations confirm quantitatively the previous qualitative interpretation of the data. Holes diffusing back to the high field region from the bulk do generate for delayed avalanches.

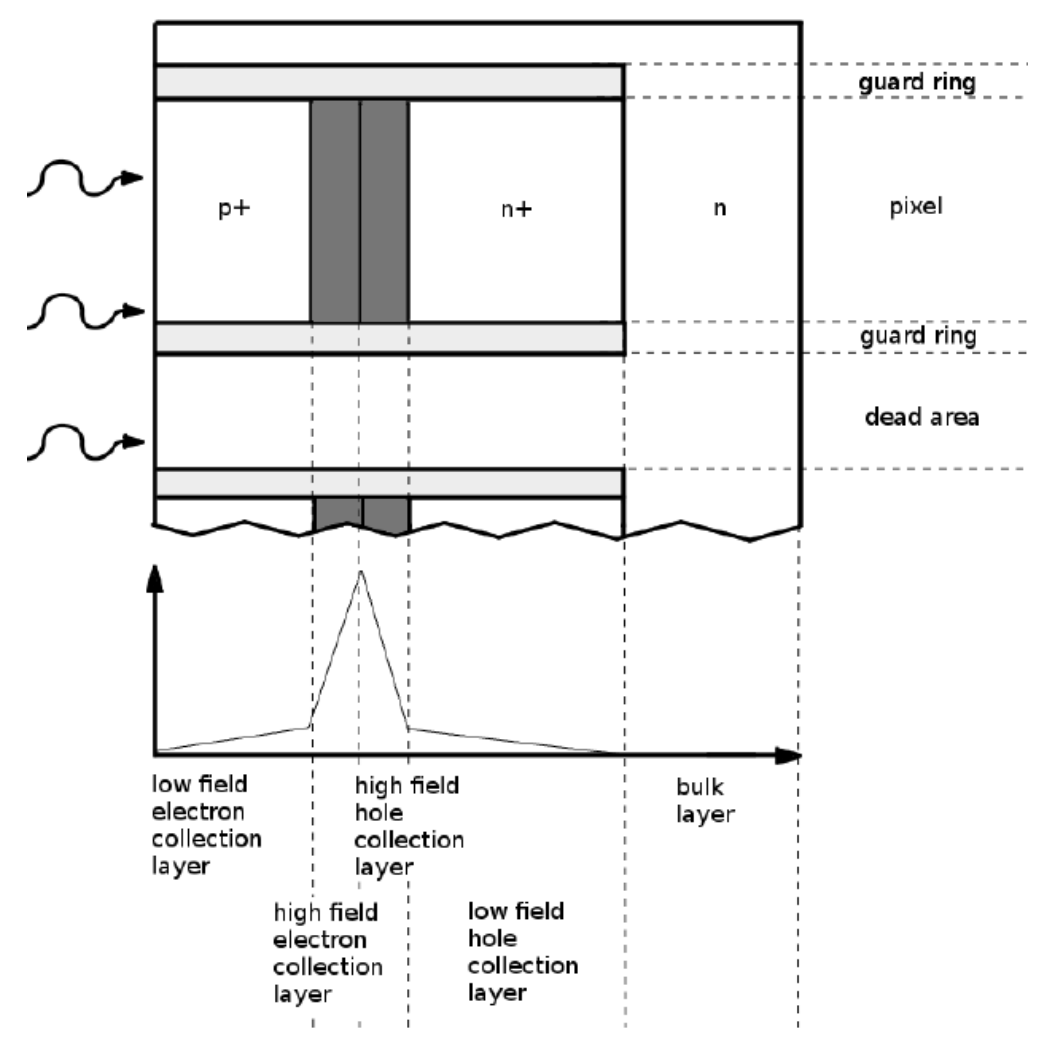

Figure 5. Schematic drawing of the junction structure used in the simulations.

\section{Consequences for correlated avalanches}

Can this phenomenon be responsible for after-pulsing, or at least for some of the afterpulses? We have to rely on simulations again to make a quantitative statement. The spectrum of photons produced in the avalanches was measured in [8]. The source of photons is now a point at the p-n junction emitting photons isotropically. The avalanche location was sampled uniformly across the active surface. Refractions at the silicon-SiO2, SiO2-epoxy and Epoxy-air interfaces were accounted for. A significant fraction of the photons emitted towards the air are indeed reflected back into the MPPC. The effect of micro-cell recovery was accounted for by reducing the avalanche triggering efficiency with a 13ns time constant.

The timing distribution of correlated avalanches due to optical photons produced in the parent avalanche is shown in Figure 7. Avalanches due to optical photons absorbed in the high field region of a neighbouring micro-cell populate the first bin. The filled circles show the default simulations using the optical photon yield measured in [2]. The open circles were rescaled by a factor of 2 in order to match the simulated cross-talk (prompt) rate with the one measured in [8]. After-pulses are clearly visible in Figure 7. They are heavily suppressed due to recovery in the first 10ns. In [2], after-pulsing was parameterized by two exponential time constants and corresponding rate (calculated as the number of after-pulsing avalanches per 
single parent avalanche). The red line shown in Figure 7 is calculated using these 4 parameters. Recovery is not accounted for in this calculation, hence the red line is only valid above $20 \mathrm{~ns}$.

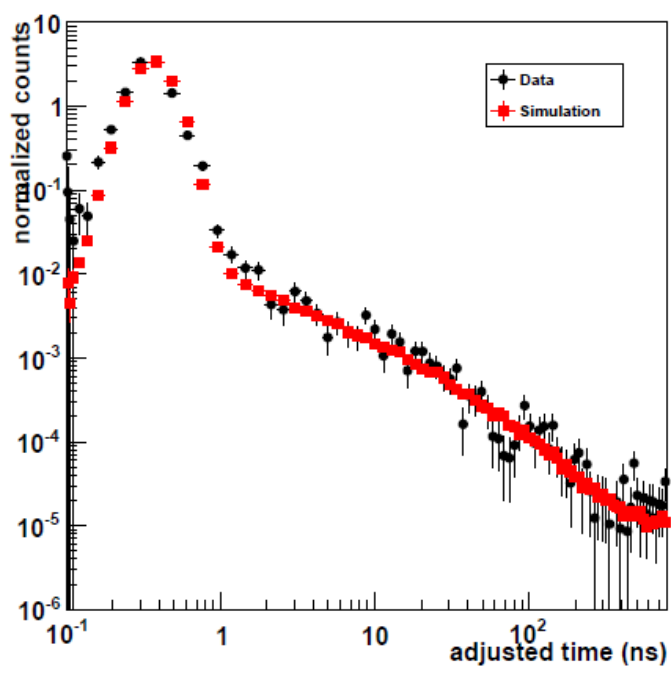

Figure 6. Comparison of data and simulations for $637 \mathrm{~nm}$ at $-60 \mathrm{C}, 1.9 \mathrm{~V}$ over-voltage.

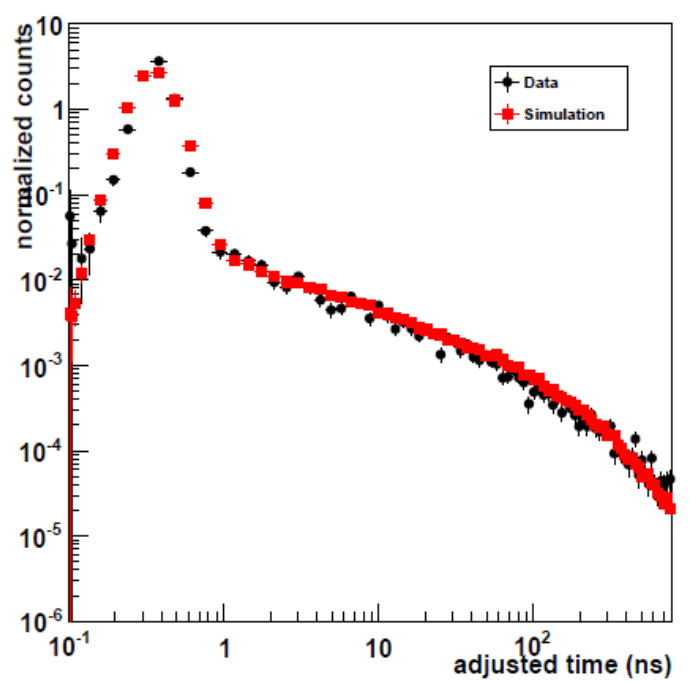

Figure 7. Comparison of data and simulations for $820 \mathrm{~nm}$ at $-60 \mathrm{C}, 1.9 \mathrm{~V}$ over-voltage.

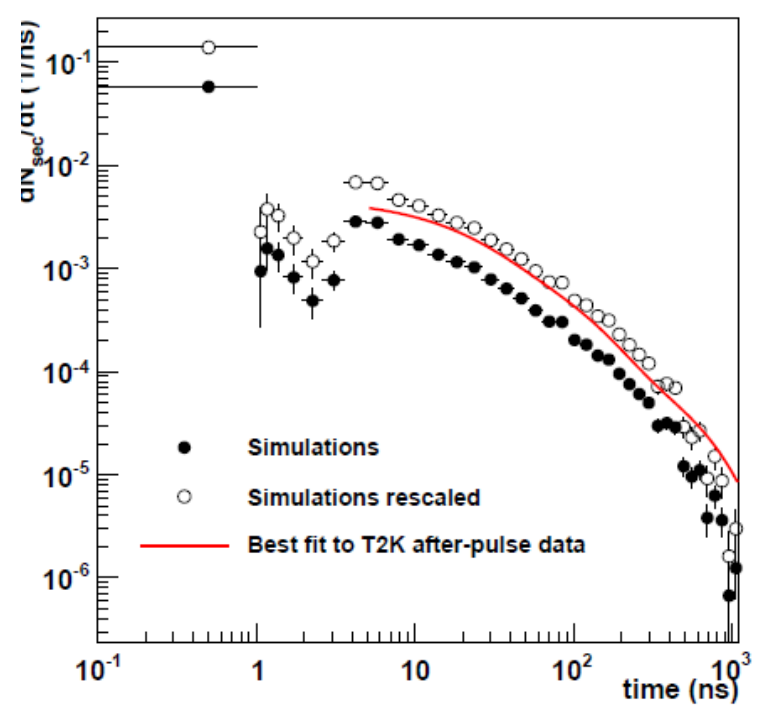

Figure 8. Timing distribution of correlated avalanches due to optical photons produced in the parent avalanche. Comparison of the data (line) and simulations (symbols).

The data agree qualitatively with the rescaled simulations. There are several uncertainties that can explain the differences: quality of fit to after-pulsing data, uncertainties on measured photons flux, and uncertainties in operating voltage. Nevertheless, we can conclude that a significant fraction of after-pulse avalanches is due to optical photon produced in the parent avalanche and absorbed in the Silicon bulk. 


\section{Summary and outlook}

It has been known for a while that cross-talk can be minimized by having a buried layer located below the junction to prevent charge carrier from the bulk from entering the high field region. Our results demonstrate that such a layer would enhance the MPPC performances reducing after-pulsing and probably the dark noise rate as well. Indeed it is likely that some dark noise avalanches are due to holes thermally created in the Silicon bulk. Reducing the correlated avalanche rate should then allow running at higher over-voltage possibly improving the photodetection efficiency and timing resolution. Hamamatsu photonics is working on a new design including a buried layer that is expected to be available by early 2013. Furthermore, SiPM manufacturers should seriously consider producing devices with a narrow charge collection region (1-3 $\mu \mathrm{m}$ deep) that would only be sensitive to Ultra-violet and blue light but would have lower dark noise and correlated avalanche rates.

\section{Acknowledgements}

We thank Hamamatsu Photonics Inc. and especially Yuji Iwai for motivating this work and providing the $820 \mathrm{~nm}$ laser system. And, we thank Colleen Gihuly for carefully reviewing the manuscript.

\section{References}

[1] Hamamatsu MPPC. http://sales.hamamatsu.com/en/products/solid-state-division/si-photodiodeseries/mppc.php

[2] A. Vacheret et al., "Characterization and Simulation of the Response of Multi Pixel Photon Counters to Low Light Levels", Nucl. Instrum. and Meth. vol. A656 p. 69 (2011).

[3] P. Eckert, H-C. Schultz-Coulon,W. Shen, R. Stamen and A. Tadday, Nucl. Instrum. and Meth. A 620, p.217 (2010).

[4] A. Ronzhin et al., "Tests of timing properties of silicon photomultipliers", Nucl. Instrum. and Meth A616, p.38 (2010).

[5] Y. Du and F. Retière, Nucl. Instrum. and Meth. A 596, p.396 (2008)

[6] P. Buzhan, B. Dolgoshein, A. Ilyin, V. Kaplin, S. Klemin, R. Mirzoyan, E. Popova, and M. Teshima, "The cross-talk problem in SiPMs and their use as light sensors for imaging atmospheric Cherenkov telescopes", Nucl. Instrum. and Meth. vol. A610, p. 131-134 (2009)

[7] S. Cova, A. Lacaita, and G. Ripamonti, "Trapping phenomena in avalanche photodiodes on nanosecond scale", IEEE Electron Device Lett., vol. 12, pp. 685-687 (1991)

[8] R. Mirzoyan, R. Kosyra and H.-G. Moser, "Light emission in Si avalanches", Nucl. Instrum. and Meth., A610, p.98 (2009).

[9] H. Oide, T. Murase, H. Otono, and S. Yamashita, "Studies on multiplication effect of noises of PPD, and a proposal of a new structure to improve the performance", Nucl. Instrum. and Meth A623, p.324 (2010)

[10] T. Nagano, T. Baba, K. Sato, and K. Yamamoto, "Timing Resolution Improvement of MPPC for TOF-PET Imaging", Presented at IEEE NSS/MIC N22-2 